Regards sur l'économie allemande

Bulletin économique du CIRAC

$100 \mid 2011$

Varia

\title{
Analyse. « Réforme » de Hartz IV : un compromis coûteux
}

Isabelle Bourgeois

\section{OpenEdition}

Édition électronique

URL : http://journals.openedition.org/rea/4230

DOI : $10.4000 /$ rea. 4230

ISBN : 978-2-8218-0894-2

ISSN : 1965-0787

\section{Éditeur}

CIRAC

Édition imprimée

Date de publication : 17 mars 2011

Pagination : 51-53

ISSN : 1156-8992

\section{Référence électronique}

Isabelle Bourgeois, «Analyse. "Réforme » de Hartz IV : un compromis coûteux », Regards sur l'économie allemande [En ligne], 100 | mars 2011, mis en ligne le 19 septembre 2011, consulté le 15 septembre 2020. URL : http://journals.openedition.org/rea/4230

Ce document a été généré automatiquement le 15 septembre 2020

(c) CIRAC 


\title{
Analyse. « Réforme » de Hartz IV : un compromis coûteux
}

\author{
Isabelle Bourgeois
}

1 Le 25 février, après d'interminables négociations et une très houleuse procédure de conciliation, le Bundesrat a définitivement adopté la «Loi sur le calcul des besoins de base et amendant les Chapitres 2 et 12 du Code social» (Gesetz zur Ermittlung von Regelbedarfen und zur Änderung des 2. und 12. Buches Sozialgesetzbuch).

\section{Un compromis 'à l'allemande'}

2 Ce compromis cherche à concilier les intérêts budgétaires du Bund, des Länder et des communes, les intérêts respectifs de l'opposition et du gouvernement fédéral, comme ceux des différents partis en campagne électorale (7 échéances !). C'est cette complexité des équilibres à trouver qui avait prolongé les négociations au-delà du 31 décembre. Cette date butoir avait été fixée par le Tribunal constitutionnel fédéral dans un arrêt du 9 février 2010. Considérant que le mode de calcul du forfait mensuel de base (Regelsatz) versé aux bénéficiaires du revenu de substitution prévu par le chapitre II du Code social (SGB II) depuis la loi Hartz IV s'assimilait à une "estimation 'à l'aveuglette' ", la Cour avait fait injonction aux législateurs de définir des critères argumentés et transparents pour calculer le montant de ce forfait. Et, rappelant que «les enfants ne sont pas des adultes miniatures", elle avait en particulier exigé qu'il soit tenu compte des besoins réels des enfants scolarisés, notamment en ce qui concerne le développement de leur personnalité (voir REA 95/10).

\section{Un accord en deux volets}

3 L'accord conclu le 25 février se compose dès lors de deux volets. Le premier redéfinit le montant du forfait de base pour les adultes et prévoit un "paquet éducation/ participation » (Bildungs- und Teihabepaket) pour les enfants ; c'est là le contenu du texte de loi voté en dernière lecture par le Bundesrat. Le deuxième volet rassemble, sous la 
forme de procès-verbaux en annexe du texte de la loi, une série de décisions connexes qui donneront lieu à négociation séparée. Sortes de conditions préalables à un accord, elles constituent la base du compromis par-delà les clivages politiques.

\section{Une loi...}

La loi du 25 février amende le chapitre SGB II en décidant :

\section{... qui crée de nouvelles prestations pour les enfants...}

la création de "prestations éducation/participation » : contribution aux fournitures scolaires, frais de cantine et de soutien scolaire, aux excursions scolaires et aux activités musicales ou sportives. Les communes affecteront des travailleurs sociaux en milieu scolaire. Ces dispositions, immédiatement entrées en vigueur, s'appliquent aux enfants vivant sous le toit d'un bénéficiaire de Hartz IV etd'une personne à faibles revenus ou percevant une allocation logement (environ 2,5 millions d'enfants au total) ;

\section{... révise à la hausse le forfait de base...}

la révision du forfait de base de Hartz IV (près de 5 millions de personnes: les bénéficiaires de ce revenu de transfert et les membres de leur famille ; voir REA 97/10). Avec effet rétroactif au $1^{\mathrm{er}}$ janvier 2011, le forfait mensuel de base est augmenté de $5 €$ et porté à $364 €$. Une nouvelle hausse de $3 €$ interviendra au $1^{\mathrm{er}}$ janvier 2012, et le forfait sera ensuite à nouveau révisé en fonction de l'inflation et de l'évolution des salaires. Par ailleurs, le revenu salarié n'est plus totalement défalqué du revenu de transfert. Quant à "l'indemnité sociale " (Sozialgeld) que touchent les enfants, son montant mensuel reste inchangé ( $215 €$ pour les moins de 6 ans, $251 €$ pour les 6-13 ans et $287 €$ pour les 14-18 ans), puisque sont venus s'y ajouter les nouvelles prestations éducation/ participation ;

\section{... et modifie la répartition du financement entre Bund, Länder et communes}

une nouvelle répartition des coûts induits entre Bund, Länder et communes. Etant donné que la gestion des prestations éducation/participation relève des compétences des communes, le Bund leur affecte à ce titre un montant forfaitaire de 1,6 milliard $€$ par an ; il leur verse en outre un montant total de 400 millions $€$ sur la période 2011/13 pour couvrir le surcroît de dépenses induit par la gratuité des cantines et l'affectation de travailleurs sociaux en milieu scolaire. A partir de 2013, ce versement compensatoire sera fonction des dépenses réelles engagées par les communes. Par ailleurs, le texte fixe jusqu'à 2013 une nouvelle clé de répartition entre Bund et Länder pour le financement des allocations logement et chauffage versées aux bénéficiaires de Hartz IV. 


\section{Et des procès verbaux en annexe}

8 Le second volet (procès verbaux d'accords figurant en annexe de la loi) prévoit essentiellement deux dispositions à adopter ultérieurement :

\section{Le Bund financera le minimum vieillesse}

la première (modification du SGB XII dédié àl'aide sociale), qui avait longtemps été une pomme de discorde entre Bund, Länder et communes, modifie le financement du minimum vital versé aux personnes âgées ou dans l'incapacité d'occuper un emploi (Grundsicherung im Alter und bei Erwerbsminderung): le Bund en supportera le coût intégral en 2014. Pour ce faire, il ne versera plus à l'Agence fédérale pour l'emploi que l'équivalent d'un demi-point de pourcentage de l'encours de la TVA au lieu du point qui lui était affecté depuis la hausse de la TVA en 2007 ;

\section{Adoption de divers SMIC légaux}

Les deux autres, qui entreront en vigueur le $1^{\mathrm{er}}$ mai, étaient la condition sine qua non du vote de la loi par l'opposition : l'introduction d'un salaire minimum légal d'une part dans les services de sécurité (dont les transports de fonds) et la formation continue via l'extension du domaine d'application de la Loi sur les travailleurs détachés (Arbeitnehmer-Entsendegesetz, AEntG), d'autre part dans l'intérim, par amendement de la Loi sur la cession des salariés (Arbeitnehmerüberlassungsgesetz, AÜG).

\section{Un compromis boîteux}

Certes, les termes de ce compromis ont permis, comme souvent dans l'histoire parlementaire allemande, de mettre fin à l'obstruction du Bundesrat où domine actuellement l'opposition. Ironie du destin, il a été voté dans des conditions très semblables à celles de la loi qu'il 'réforme' : le vote de la loi Hartz IV avait été lui aussi plus que houleux et n'était intervenu qu'in extremis avant la fin de l'année parlementaire 2005. Mais le compromis adopté en 2011 est boîteux à plusieurs égards, et risque d'avoir des effets néfastes sur le marché de l'emploi, de même que sur les budgets sociaux.

\section{Retour en arrière larvé sur le caractère forfaitaire de l'indemnité de base}

13 Il fait fi des injonctions de la Cour de Karlsruhe qui exigeait 'simplement' que soient définis des critères transparents pour le calcul du forfait de base; elle n'avait pas pris position sur le montant en tant que tel (voir REA 95/10). La Cour demandait donc que soit dressé un catalogue chiffré des besoins concrets que doit couvrir la collectivité au titre de la solidarité. Mais ce faisant, elle ravivait le conflit cornélien entre le socialement souhaitable et l'avenir durable de l'Etat-Providence. Et de fait, au fil des 
négociations, le travail des législateurs s'est réduit comme peau de chagrin à la seule indexation du forfait de base sur le coût de la vie - et des salaires ; or ce dernier point est en totale contradiction avec l'esprit même de la Loi Hartz IV qui visait justement à rompre avec la trappe à inactivité de l'ancien régime d'aide aux chômeurs en fins de droits : sa base de calcul était le salaire antérieur (voir REA 68/04).

\section{SMIC légal dans l'intérim : retour en force du socialement souhaitable...}

Si le caractère 'bâtard' de l'actuel compromis reflète à l'évidence la surenchère sociale des partis en cette 'super-année électorale', il révèle encore plus l'ampleur de la rupture provoquée au sein de l'actuelle opposition par le dispositif phare de l'Agenda 2010 mis en œuvre par l'ancienne coalition SPD/Verts (voir dans ce numéro la contribution de R. Lasserre). Depuis l'entrée en vigueur de la Loi Hartz IV en 2005, SPD, Verts et Die Linke ne cessent de chercher à revenir en arrière sur ce qui est à leurs yeux le péché capital du gouvernement Schröder II : le « démantèlement » de l'Etat social. La Cour de Karlsruhe leur offrait là l'occasion idéale pour tenter de l'effacer, ainsi qu'un autre 'péché' encore commis par ce chancelier: l'assouplissement du régime de l'intérim dans le cadre de la politique générale de flexibilisation de l'emploi. Or le Tribunal fédéral du Travail venait d'invalider le 14 décembre les conventions collectives du secteur. Faute de pouvoir abroger HartzIV, le compromis aura donc permis à la gauche de réaliser une de ses revendications phares : l'institution d'un SMIC légal dans le travail intérimaire (voir REA 86/08).

\section{... au détriment des actifs les moins qualifiés}

Certes, le SMIC légal dans l'intérim ne concerne que moins d'un million de personnes, mais elles jouent un rôle clé dans l'activité outre-Rhin, où le travail temporaire est majoritairement choisi par des actifs qualifiés, en adéquation avec la demande de l'industrie. Cette forme d'emploi flexible avait permis aux entreprises de réagir aux signes avant-coureurs de reprise après la récession et contribué à stabiliser le marché de l'emploi. Par leur qualification, les intérimaires compensent actuellement le manque d'ingénieurs et techniciens. Or ceux-ci sont pour la plupart sous contrat chez les géants du secteur, qui approuvent d'ailleurs un seuil salarial élevé valable pour la durée de la cession et aussi celle des formations ('inactivité'). Mais les moins qualifiés et les chômeurs en réinsertion sont employés par les petites sociétés. Autrement dit, comme dans le cas du SMIC postal (voir REA 85/08), le SMIC dans l'intérim force la cartellisation du marché et accroît la cohorte des outsiders - les moins qualifiés que les réformes Schröder cherchaient à réintégrer dans le marché du travail.

\section{SMIC légal dans la sécurité : une mesure protectionniste}

Quant à la création d'un SMIC légal dans les services de sécurité, le choix de l'extension de la Loi sur les travailleurs détachés (AEntG) qui avait été adoptée en 1996 pour protéger les salariés du BTP est-allemand de la concurrence est-européenne (voir REA 
88/08) confirme qu'il s'agit d'ériger des barrières « anti-dumping salarial » : le $1^{\mathrm{er}}$ mai, la Directive Services entrera pleinement en vigueur. Or celle-ci est ardemment combattue depuis 2005 par la gauche allemande qui en a fait son argument principal pour revendiquer un SMIC légal généralisé dans les services (voir REA 71/05).

\section{Quelle cohérence politique?}

17 Enfin, en termes de politique économique, sociale et budgétaire, le coût de cette "réforme " soulève un certain nombre de questions. Alors que, depuis la hausse de 3 points de la TVA en 2007, un point était consacré à la baisse des charges patronales (les cotisations chômage sont passées de 6,5 \% à 4,2 \%), l'affectation de 50 \% de cet encours au minimum vieillesse soulève la question de l'équilibre budgétaire à terme de l'Agence de Nuremberg (ses missions sont comparables à celles de Pôle Emploi en France); ses recettes se voient réduites d'autant. S'y ajoute le coût de l'augmentation régulière du forfait et des nouvelles prestations éducation/participation. L'Agence ayant épuisé ses réserves durant la récession, son déficit est donc programmé. Le Bund devra alors le compenser soit en recourant à la dette soit en décidant une hausse des prélèvements, soit encore en réduisant le niveau des prestations. Les périodes électorales sont rarement bonnes conseillères...

\section{INDEX}

Mots-clés : Hartz IV, allocation chômage, politique sociale, réforme, enfant, famille, financement, revenu, niveau de vie, salaire minimum, politique de l'emploi 